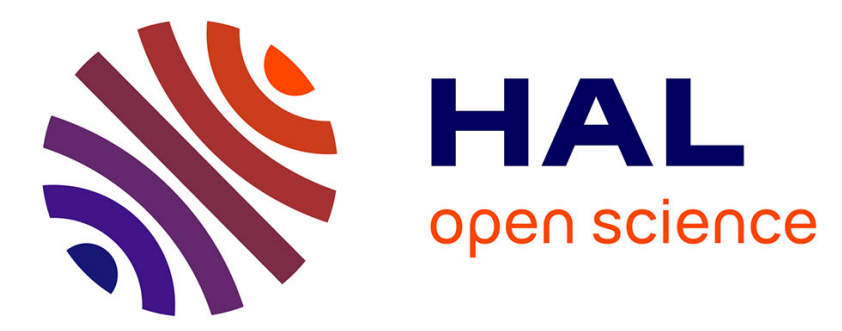

\title{
Cotes et crues de protection, de sûreté et de danger de rupture
}

\author{
P. Royet, G. Degoutte, L. Peyras, J. Lavabre, F. Lemperrière
}

\section{To cite this version:}

P. Royet, G. Degoutte, L. Peyras, J. Lavabre, F. Lemperrière. Cotes et crues de protection, de sûreté et de danger de rupture. Colloque CFBR-SHF: Dimensionnement et fonctionnement des évacuateurs de crues, Jan 2009, Paris, France. 8 p. hal-00468565

\section{HAL Id: hal-00468565 https://hal.science/hal-00468565}

Submitted on 31 Mar 2010

HAL is a multi-disciplinary open access archive for the deposit and dissemination of scientific research documents, whether they are published or not. The documents may come from teaching and research institutions in France or abroad, or from public or private research centers.
L'archive ouverte pluridisciplinaire HAL, est destinée au dépôt et à la diffusion de documents scientifiques de niveau recherche, publiés ou non, émanant des établissements d'enseignement et de recherche français ou étrangers, des laboratoires publics ou privés. 


\title{
COTES ET CRUES DE PROTECTION, DE SURETE ET DE DANGER DE RUPTURE
}

\section{Protection, safety and danger levels and associated floods}

\author{
Paul Royet, Gérard Degoutte, Laurent Peyras, Jacques Lavabre \\ Cemagref - Unité de Recherche Ouvrages hydrauliques et Hydrologie \\ 3275, Route de Cézanne - CS 40061 - 13182 Aix en Provence Cedex 5 \\ Tél: +33 (0)4 426699 42, Fax: +33 (0)4 426688 65, e-mail: prenom.nom@cemagref.fr \\ François Lemperrière \\ Hydrocop \\ 18, rue de Bourgogne - 92191 Meudon \\ Tél: +33 (0)1 453442 89, Fax: +33 (0)1 450172 06, e-mail: hydrocoop.fr@,free.fr
}

\begin{abstract}
Cette communication présente une synthèse des différentes cotes et crues de référence habituellement utilisées pour l'évaluation de la sécurité d'un ouvrage hydraulique, y compris dans le cas d'un barrage écrêteur de crues ou d'une digue de protection contre les inondations. Les discussions au sein du groupe de travail du CFBR consacré à la justification de la stabilité des barrages et les travaux de recherche menés au Cemagref dans le domaine de l'analyse de risques appliquée aux ouvrages hydrauliques montrent les limites de ces définitions et ouvrent de nouvelles perspectives.

Des propositions sont donc faites pour clarifier la terminologie. Cela aboutit à privilégier la détermination des cotes atteintes dans la retenue pour différents scénarios de crues. Au final, il apparaît possible d'associer des probabilités d'occurrence au dépassement de la cote de sûreté ou de la cote de danger de rupture, et parallèlement de proposer des valeurs minimales cibles pour ces probabilités, en fonction des types d'ouvrages et de leur classe.
\end{abstract}

This paper presents a synthesis of different level and flood references usually used for assessing the safety of hydraulic structures, including dams for flood control or dikes for flood protection. Discussions in the FrCOLD working group on justification for the stability of dams and research conducted by Cemagref in the field of risk analysis applied to hydraulic works show the limits of these definitions and open new perspectives.

Proposals are made to clarify terminology. This leads to emphasize the determination of levels reached in the reservoir for different scenarios of flooding. Then it is possible to associate probabilities of occurrence to the maximum water level and to the level of danger of rupture, and parallel to propose minimum targets for these probabilities, depending on the types of hydraulic works and their class.

\section{INTRODUCTION}

Pour l'évaluation de la sécurité d'un ouvrage hydraulique vis-à-vis des crues, on utilise couramment différentes notions qu'il s'avère intéressant de préciser :

- la cote normale d'exploitation ou « retenue normale» $(\mathrm{RN})$;

- la crue de protection d'un ouvrage destiné à limiter l'impact des crues en aval ;

- la cote des plus hautes eaux (PHE), associée à la « crue de projet» [1] ;

- la cote de danger.

Si certaines cotes sont fixes et intrinsèques à l'ouvrage (cote RN d'un barrage, cote de protection par une digue), d'autres peuvent évoluer en fonction de la connaissance de l'hydrologie ou de la sécurité structurelle de l'ouvrage.

Nous proposons dans cette communication une clarification de ces différentes notions, et présentons des résultats de travaux de recherche qui ouvrent de nouvelles perspectives pour l'évaluation de la sécurité des barrages et des digues vis à vis des crues. Cela concerne les ouvrages existants ou en projet. 


\section{COTE NORMALE D'EXPLOITATION D'UN BARRAGE}

Egalement dénommée cote de retenue normale $(\mathrm{RN})^{1}$, il s'agit de la cote « habituelle » ou « normale » de remplissage du barrage, correspondant au niveau autour duquel le plan d'eau se situe une grande partie de l'année ${ }^{2}$. Pour les barrages équipés d'un évacuateur à seuil libre, c'est la cote de ce seuil. Dans le cas de seuils libres multiples, c'est la cote du seuil le plus bas. Pour les évacuateurs vannés, cette cote correspond par exemple à la cote supérieure d'un clapet en position relevée ou au bordé supérieur d'une vanne segment abaissée. Dans tous les cas, il s'agit d'une cote qui est physiquement matérialisée par un organe du barrage.

Cette cote a valeur réglementaire et figure dans l'arrêté d'autorisation ou le décret de concession du barrage. C'est elle qui permet de déterminer le volume de la retenue $\mathrm{V}$, qui a également valeur réglementaire en particulier pour définir la classe de l'ouvrage au vu de la récente réglementation française.

\section{COTE ET CRUE(S) DE PROTECTION}

Ces notions ne concernent que les barrages écrêteurs de crues et les digues de protection contre les inondations.

Un barrage écrêteur de crues est équipé de pertuis (libres ou vannés), dont le dimensionnement est basé sur la capacité du lit en aval. On évalue le débit maximal non dommageable du cours d'eau en aval du barrage (par exemple débit de plein bord du lit mineur ou débit d'un ouvrage aval créant section de contrôle) et on dimensionne les pertuis pour délivrer au maximum ce débit. Si le débit entrant dans la retenue lui est supérieur, il y a stockage temporaire dans la tranche d'eau de laminage, jusqu'à atteindre la cote d'un déversoir de surface dont la fonction est d'assurer la sécurité du barrage lors des événements extrêmes

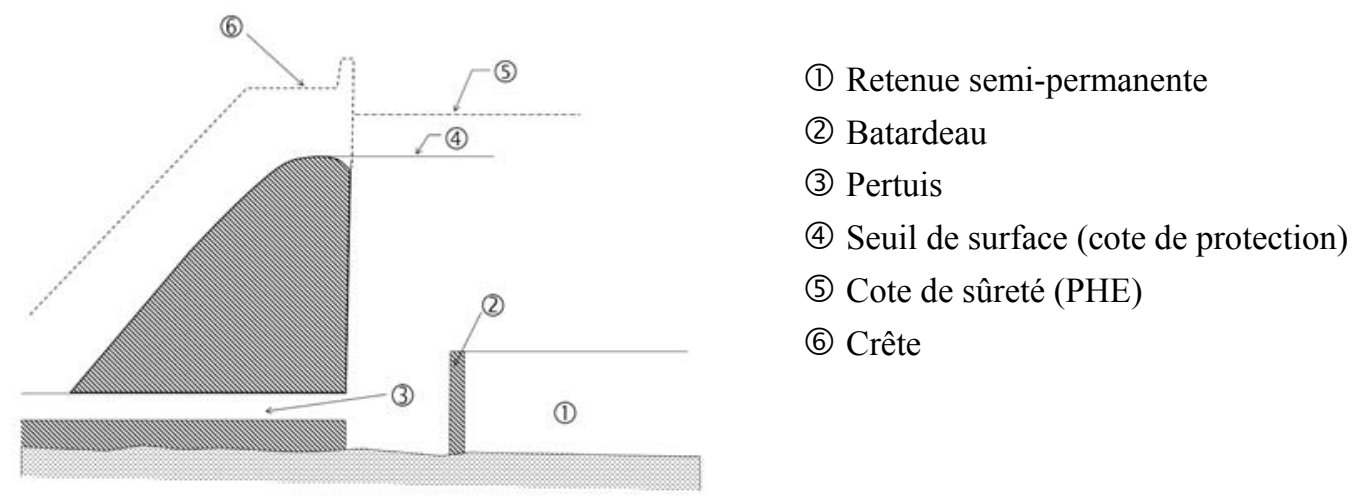

Figure 1: coupe schématique d'un barrage écrêteur de crues.

Pour les digues de protection contre les inondations, le raisonnement proposé est semblable : la digue protège la plaine de l'inondation pour les crues courantes. Mais pour les événements rares à exceptionnels, c'est la rupture de la digue par surverse dont on souhaite se prémunir. A cette fin, la digue est équipée d'un déversoir ou aménagée pour être rendue déversante sur une partie de sa longueur. Lorsque cette cote est atteinte, l'inondation de la plaine va se faire de façon maitrisée ; la montée lente du niveau de l'eau dans la plaine va ensuite limiter les conséquences d'une éventuelle rupture brutale de la digue par surverse [4].

On peut donc proposer les définitions suivantes :

- Cote de protection :

- cote du déversoir de surface d'un barrage écrêteur de crues ;

- cote du déversoir de sécurité d'une digue ou cote de la partie de la digue aménagée pour résister à la surverse (cote le cas échéant variable de l'amont à l'aval).

Il s'agit d'une donnée intrinsèque à l'ouvrage.

A cette cote est associée une crue ou une famille de crues qui, vu la présence de l'ouvrage, ne seront pas dommageables pour les enjeux en aval du barrage ou dans le val protégé par la digue.

\footnotetext{
${ }^{1}$ Le terme anglais est : Normal Water Level (NWL)

${ }^{2}$ Sachant toutefois que la cote $\mathrm{RN}$ est, sur certains ouvrages à fonctionnement fortement saisonnier, peu fréquemment atteinte au cours de l'année.
} 
- Crue(s) de protection ${ }^{3}:$ crue(s) provoquant un remplissage jusqu'à la cote de protection et pour laquelle on obtient l'optimum d'efficacité vis-à-vis de l'objectif de protection à l'aval ou dans le val.

Aussi bien pour les barrages écrêteurs que pour les digues, la probabilité annuelle d'occurrence associée à cette famille de crues est habituellement de $10^{-1}$ à $10^{-2}$ selon les enjeux à protéger, soit une période de retour de 10 à 100 ans.

\section{COTE ET CRUE(S) DE SURETE}

Ces notions concernent tous les barrages et nous proposons de les appliquer aussi aux digues de protection contre les inondations.

Pour un barrage au stade du projet, la démarche habituelle consiste à déterminer la crue ${ }^{4}$ associée à une période de retour qui dépend du niveau de sécurité exigé (par exemple 100 à 2000 ans pour un barrage de classe $\mathrm{D}$ ou $\mathrm{C}^{5}, 1000$ à 5000 ans pour un grand barrage en béton et 10000 ans pour un grand barrage en remblai) ; on calcule la cote maximale atteinte par le plan d'eau en fonction des capacités de l'évacuateur de crues, tout en tenant compte éventuellement de l'écrêtement des hydrogrammes. Les calculs sont classiquement menés sous une double hypothèse :

- crue arrivant sur une retenue remplie à la cote normale $(\mathrm{RN})$;

- fonctionnement nominal sans défaillance des organes d'évacuation.

On obtient ainsi ce que l'on dénomme la cote des plus hautes eaux (PHE), cote qui est reprise dans l'arrêté d'autorisation ou le décret de concession de l'ouvrage et prend ainsi une valeur réglementaire. Au-delà de la cote des PHE, un calcul de revanche pour la protection contre l'effet des vagues amène à déterminer la cote de la crête, et celle d'un éventuel pare-vagues.

Pour un barrage en service, nous proposons la démarche inverse, développée par Peyras et al. dans une communication à ce même colloque [7]. Connaissant la cote réglementaire des PHE, ou l'ayant recalculée à partir de la revanche nécessaire, on va rechercher la probabilité associée aux crues susceptibles de provoquer cette cote de retenue, en tenant compte éventuellement du remplissage variable du réservoir en début de crue.

La même démarche peut s'appliquer aux digues de protection contre les inondations. A partir de la connaissance du profil en long de la crête et d'un calcul de revanche nécessaire pour se protéger des vagues ou des surcotes dans les courbes, on peut calculer un profil de ligne d'eau admissible vis-à-vis de considérations liées à la sécurité hydraulique de la digue. A ce profil peut être associée une période de retour par comparaison avec les lignes d'eau dans la rivière en crues.

Ainsi nous pouvons proposer les définitions suivantes :

- Cote de sûreté (PHE) : cote de la retenue (cas des barrages) ou de la rivière (cas des digues) lors des crues, laissant encore une revanche pour se protéger de l'effet des vagues.

A cette cote, l'ouvrage doit répondre à tous les standards de sécurité, que ce soit sur le plan structural (résistance au cisaillement ou au glissement, résistance en fondation, résistance à l'érosion interne) ou sur le plan hydraulique (pas de débordement des coursiers, ouvrages de dissipation correctement dimensionnés). Et l'on dispose encore de marges avant d'atteindre des états-limite de rupture.

La probabilité annuelle de dépassement associée à cette cote dépend des classes d'ouvrages et des enjeux menacés en cas de rupture; les ordres de grandeur sont les suivants : $10^{-2}$ à $10^{-3}$ pour les digues, $10^{-2}$ à $10^{-4}$ pour les barrages. Pour les barrages, la probabilité correspond à des recommandations qui ont quasiment valeur de prescriptions : pratiques du CTPBOH ${ }^{6}$, recommandations du CFBR.

- Crue(s) de sûreté ${ }^{7}$ : crue(s) provoquant un remplissage jusqu'à la cote PHE.

Nous ajoutons volontairement un « $\mathrm{S}$ » à crue, car le remplissage à la cote des PHE peut résulter de divers événements hydrologiques, depuis la crue courte présentant un débit de pointe très élevé (donc bien écrêtée par la retenue) jusqu'à la crue longue présentant un débit de pointe durablement élevé (écrêtement très

\footnotetext{
${ }^{3}$ Le terme anglais est : Protection design flood(s) [3]

${ }^{4}$ Habituellement dénommée « crue de projet », « design flood » en anglais [1]

${ }^{5}$ Référence [2] du CFBR, page 25.

${ }^{6} \mathrm{CTPB}$ : Comité technique permanent des barrages et des ouvrages hydrauliques

${ }^{7}$ Inflow design flood [3]
} 
réduit) en passant par les crues à pointes multiples (écrêtement de la première pointe, mais pas des suivantes). C'est ce que mettent en évidence Lavabre \& al. [6]. De même, les crues peuvent arriver sur une retenue partiellement vide, accroissant ainsi la capacité d'écrêtement.

Il est aussi possible d'envisager des situations de défaillance de l'évacuateur de crues, par exemple le blocage d'une ou plusieurs vanne(s) ou l'obstruction d'une ou plusieurs passe(s) par des débris. A de telles défaillances, peuvent être associées leurs probabilités d'occurrence. Elles peuvent dans certains cas être liées à la probabilité de l'événement hydrologique incident. Par exemple, la probabilité d'obstruction par les flottants d'une passe de dimensions réduites va être faible pour les crues courantes et devenir plus importante dès les crues rares qui ont tendance à «nettoyer» le cours d'eau et ses berges. De telles combinaisons d'événements impliquant une modification de la débitance de l'évacuateur de crues vont conduire à différentes cotes dans la retenue auxquelles il sera possible d'associer une probabilité d'occurrence qui ne sera plus forcément celle de la crue incidente.

\section{COTE ET CRUE(S) DE DANGER DE RUPTURE}

Ces notions concernent barrages et digues.

- Cote de danger de rupture : cote au-delà de laquelle l'ouvrage risque de subir des dégâts majeurs pouvant conduire rapidement à la rupture. L'atteinte de cette cote constitue un état-limite ultime pour l'ouvrage.

Cette terminologie nous parait plus précise que la terminologie «cote de danger» utilisée jusqu'à maintenant pour les plans particuliers d'intervention (PPI) et nous proposons de la généraliser à tous les ouvrages hydrauliques. Mais il s'agit de la même chose, à savoir la cote correspondant à "l'état de péril imminent pris lorsque l'exploitant estime qu'il n'a plus le contrôle de l'ouvrage » (arrêté du 2 février 2002). La pratique consiste à déterminer cette cote de la façon suivante :

- cote de la crête pour un remblai semi-homogène ou à masque amont ;

- cote d'arase du noyau pour un remblai zoné ;

- cote conduisant au débordement d'un coursier avec érosion du talus aval d'un remblai ;

- cote correspondant à la limite de résistance à l'effort tranchant ou à l'ouverture de fissure d'un barrage ou d'une digue poids ;

- cote pouvant être sensiblement supérieure à la crête pour un barrage voûte.

Cette cote est une donnée intrinsèque à l'ouvrage, tenant compte de ses modes de rupture et de sa sécurité structurale.

Comme pour la cote de sûreté, la probabilité annuelle de dépassement acceptable associée à la cote de danger de rupture dépend des classes d'ouvrages et des enjeux menacés en cas de rupture. Elle n'est aujourd'hui fixée par aucun texte ni aucune recommandation. Nous faisons donc la proposition suivante : la probabilité annuelle de dépassement associée à la cote de danger de rupture devrait être d'un ordre de grandeur inférieure à celle de la cote de sûreté (par exemple, si la probabilité associée à la cote des PHE est de $10^{-3}$, celle associée à la cote de danger serait de l'ordre de $10^{-4}$, soit une période de retour d'environ 10000 ans).

- Crue(s) de danger de rupture $^{8}$ : crue(s) associée(s) à l'atteinte de la cote de danger de rupture.

Il s'agit là encore d'une famille de crues, avec différentes formes d'hydrogrammes arrivant sur une retenue initialement plus ou moins remplie et combinées avec différents scénarios de défaillance des évacuateurs ou déversoirs.

\section{EXEMPLES D’APPLICATION}

\section{VI.1 Cas d'un barrage écrêteur de crues}

Il s'agit d'un barrage en remblai d'enrochements de $41 \mathrm{~m}$ de hauteur étanché par un masque amont en béton bitumineux. Le fonctionnement hydraulique du barrage (tableau 1) peut être décrit ainsi :

${ }^{8}$ Safety check flood [3] 
- une retenue semi-permanente, utilisée en été pour le soutien d'étiage, est créée en fond de cuvette par une entrée surélevée des pertuis ;

- deux pertuis non vannés $\left(18 \mathrm{~m}^{2}\right.$ de section totale) traversent le barrage à sa base; pour les crues moyennes, leur mise en charge provoque le remplissage de la retenue, tout en limitant le débit aval à $300 \mathrm{~m}^{3} / \mathrm{s}$;

- pour les crues exceptionnelles, et une fois la retenue pleine, un seuil circulaire de surface permet le déversement du débit entrant excédentaire; il donne dans deux puits puis dans une galerie de $40 \mathrm{~m}^{2}$ de section passant sous le remblai.

\begin{tabular}{|l|l|l|l|}
\hline Description & Cote & Volume à cette cote & Débit évacué \\
\hline Retenue semi-permanente (seuil des pertuis) & 242,00 & $0,9 \mathrm{hm}^{3(*)}$ & $0 \mathrm{~m}^{3} / \mathrm{s}$ \\
\hline Seuil de surface (corolle) & 261,20 & $10,8 \mathrm{hm}^{3}$ & $300 \mathrm{~m}^{3} / \mathrm{s}$ \\
\hline PHE d'origine & 266,00 & $14,7 \mathrm{hm}^{3}$ & $900 \mathrm{~m}^{3} / \mathrm{s}$ \\
\hline PHE suite à révision 1983 & 266,80 & $14,9 \mathrm{hm}^{3}$ & $920 \mathrm{~m}^{3} / \mathrm{s}$ \\
\hline Crête et cote de la route en rive gauche & 267,00 & $15,0 \mathrm{hm}^{3}$ & $920 \mathrm{~m}^{3} / \mathrm{s}$ \\
\hline
\end{tabular}

${ }^{(*)}$ volume d'eau restant disponible compte tenu de l'important envasement

\section{Tableau 1 : caractéristiques hydrauliques du barrage}

Le dimensionnement initial du barrage répondait aux principes suivants :

- le volume de la retenue permanente est déterminé en fonction des besoins pour le soutien d'étiage, ce qui définit la cote d'alimentation des pertuis ;

- la crue de période de retour 1000 ans, arrivant sur une retenue à la cote du plan d'eau permanent, devait être contenue dans le réservoir, sans déversement sur le seuil de surface ;

- la crue de période de retour 5000 ans, arrivant sur une retenue déjà pleine au niveau du déversoir, devait être évacuée avec une revanche de $1,0 \mathrm{~m}$ sous la crête.

Une première actualisation des études hydrologiques, au début des années 1980, a conduit à revoir sensiblement à la hausse les lames d'eau écoulées en crue, sans révision des débits de pointe. Avec des volumes de crue plus élevés, la cote des PHE (toujours associée à une période de retour de 5000 ans) a été portée à 266,80 $\mathrm{m}$ NGF, la revanche nécessaire de 1,0 $\mathrm{m}$ étant assurée par la présence d'un pare-vagues en bord amont de la crête. A noter qu'en rive gauche, le pare-vagues ne se poursuit pas jusqu'au versant (présence d'une route à la cote 267,00).

A la lumière d'événements récents, il a été procédé à une nouvelle actualisation des études hydrologiques. Cette dernière a confirmé les ordres de grandeur des valeurs des débits de pointe de crues des études d'origine, mais a mis en évidence une sous-estimation importante des quantiles de pluies de longue durée et une sous-estimation encore plus importante des volumes et durées de crues.

Lors de ces études hydrologiques, les méthodes SHYPRE et SHYREG mises en œuvre ont permis d'obtenir une collection d'hydrogrammes de crues dont le passage au barrage a ensuite été simulé. La méthodologie complète est décrite dans la communication de Lavabre \& al [6]. On peut ainsi obtenir la distribution de fréquence des cotes dans la retenue (figure 2).

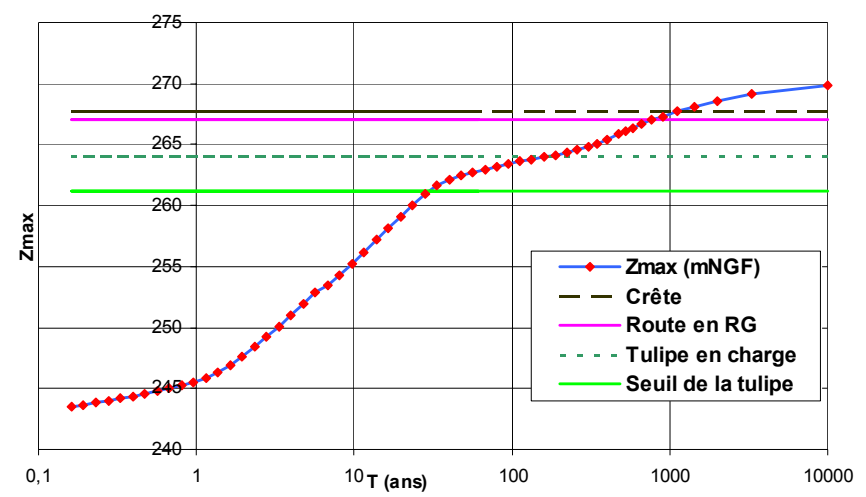

Figure 2 : Barrage écrêteur de crue. Distribution de fréquence des cotes dans la retenue 
Il ressort que le barrage n'apporte pas la protection affichée à l'origine. Le débordement sur le seuil de surface se produit en moyenne une fois tous les 30 ans, et non tous les 1000 ans comme initialement estimé. Mais plus grave, la cote réglementaire des PHE, revue à la hausse en 1983, correspond à une période de retour de 700 ans. Le débordement en rive gauche sur la route (cote 267,00) correspond à une période de retour inférieure à 1000 ans. Comme, en la situation actuelle, les flots déversés sur la route reviendraient vers le pied aval du remblai, on ne peut pas écarter le risque d'érosion du talus, voire la rupture en cas de déversement prolongé. Ce risque est présent dès que la lame d'eau déversée atteint quelques décimètres.

\section{Illustration de la terminologie proposée}

En tenant compte de la révision de l'étude hydrologique, les notions développées dans cet article se traduisent par les résultats suivants :

- cote de protection: $\quad 261,20 \mathrm{~m}$,

- cote de sûreté (PHE) : $\quad 266,80 \mathrm{~m}$,

- cote de danger de rupture : environ $267,30 \mathrm{~m}$, période de retour associée : 30 ans ;

période de retour associée : 700 ans ;

période de retour associée : 1000 ans.

La période de retour associée à l'atteinte de la cote des PHE, soit 700 ans, ne répond pas aux standards usuels qui fixent une période de retour de 10000 ans pour les grands barrages en remblai. Par ailleurs, la marge disponible, en termes de probabilité d'occurrence, entre l'atteinte de la cote de sûreté et de la cote de danger de rupture est très faible, alors que l'on attendrait que ces deux valeurs soient nettement différentes. Sur ce dernier point, ce cas illustre de manière particulièrement frappante la situation des barrages en remblai ne disposant pas d'évacuateurs à seuil libre.

Les études d'avant-projet de construction d'un évacuateur supplémentaire sur ce barrage sont en cours et, dans l'attente des travaux, des aménagements sont en train d'être apportés au PPI pour mieux anticiper le stade de péril imminent.

\section{VI.2 Exemples d'application à des barrages de stockage}

Nous reprenons (tableau 2) les exemples présentés dans l'article de Peyras \& al [7].

\begin{tabular}{|c|c|c|c|}
\hline & Barrage 1 & Barrage 2 & Barrage 3 \\
\hline Type de barrage & Remblai zoné & Remblai semi-homogène & Voûte à double courbure \\
\hline Evacuateur de crues & Déversoir à seuil libre & Déversoir à seuil libre & Evacuateurs vannés \\
\hline Surface du BV & $2 \mathrm{~km}^{2}$ & $20 \mathrm{~km}^{2}$ & $200 \mathrm{~km}^{2}$ \\
\hline $\begin{array}{c}\text { Période de retour de la crue de } \\
\text { projet d'origine }\end{array}$ & 1000 ans & 5000 ans & 5000 ans \\
\hline Usage & irrigation & eau potable & eau potable \\
\hline Marnage de la retenue & Important & Faible & Faible \\
\hline
\end{tabular}

Tableau 2 : Caractéristiques des barrages étudiés

La simulation des cotes atteintes lors des crues a été faite en tenant compte de la variabilité de la cote de remplissage de la retenue, et à partir d'une famille d'hydrogrammes de crue de différentes périodes de retour. Les éventuelles défaillances des évacuateurs de crues n'ont pas été considérées. Elles sont très improbables pour les deux déversoirs à seuil libre qui sont de dimension conséquente et non surmontés de passerelles ; pour le troisième barrage, la défaillance d'une ou des deux vannes devrait être envisagée. Cela a permis de déterminer la distribution de fréquence des cotes atteintes en crues (figure 3).
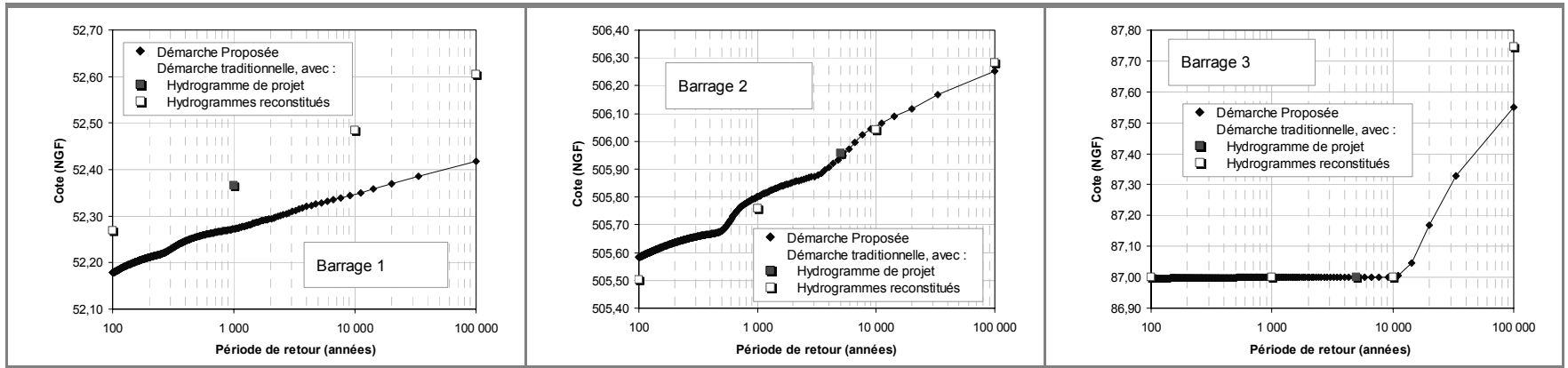

Figure 3 : Distributions de fréquence des cotes de remplissage en crue pour les trois barrages. 
Appliquées aux trois barrages, les notions développées se traduisent par les résultats suivants :

\begin{tabular}{|l|c|c|c|c|c|}
\hline & $\begin{array}{c}\text { Cote de retenue } \\
\text { normale }\end{array}$ & $\begin{array}{c}\text { Cote de sûreté } \\
\text { (PHE) }\end{array}$ & $\begin{array}{c}\text { Période de } \\
\text { retour associée }\end{array}$ & $\begin{array}{c}\text { Cote de danger de } \\
\text { rupture }\end{array}$ & $\begin{array}{c}\text { Période de retour } \\
\text { associée }\end{array}$ \\
\hline Barrage 1 & $52,00 \mathrm{~m}$ & $52,40 \mathrm{~m}$ & 60000 ans & $\begin{array}{c}53,70 \mathrm{~m}(\mathrm{sommet} \\
\text { noyau })\end{array}$ & $>>>100000$ ans \\
\hline Barrage 2 & $505,00 \mathrm{~m}$ & 506,00 & 7000 ans & $506,50 \mathrm{~m}^{(* *)}$ & $>100000$ ans \\
\hline Barrage 3 & $87,00 \mathrm{~m}$ & $87,00 \mathrm{~m}$ & 10000 ans & $90,00($ crête $+1 \mathrm{~m})$ & $>>100000$ ans \\
\hline
\end{tabular}

${ }^{\left({ }^{* *}\right)}$ La cote de la crête de ce barrage est à 507,5. Cependant, l'évacuateur de crues étant posé sur le remblai, nous considérons que la cote de danger de rupture correspond à la cote pour laquelle on observe les premiers débordements sur le coursier. La valeur proposée ici est une estimation non fondée sur des calculs de ligne d'eau

On voit donc que ces trois barrages répondent aux règles de l'art pour ce qui concerne la cote de sûreté, même si à l'origine les barrages 1 et 2 en remblai avaient été dimensionnés pour la crue de période de retour respectivement de 1000 ans et de 5000 ans.

Le barrage 1, compte-tenu de la très faible surface de son bassin versant, écrête très bien les crues et présente un niveau de sécurité tel qu'il rend envisageable une augmentation de la capacité par rehaussement du seuil; ce projet s'est très récemment concrétisé par l'installation d'un clapet dont la manœuvre saisonnière permet cette augmentation, sans réduire la sécurité en crues.

Par ailleurs, pour ces trois barrages, la période de retour associée à la cote de danger de rupture est supérieure de plus d'un facteur 10 à celle associée à la cote de sûreté.

\section{APPLICATION EN DEHORS DE LA FRANCE}

La présentation ci-dessus est basée principalement sur les barrages français. La philosophie et les propositions correspondantes semblent pouvoir s'appliquer également à d'autres pays, en tenant compte des points suivants [5] :

- $\quad$ les périodes de retour de la crue de sûreté peuvent être différentes des périodes adoptées en France ;

- $\quad$ beaucoup de futurs barrages seront dans des pays en développement sujets à des crues extrêmes très importantes, avec un coût élevé des déversoirs ;

- $\quad$ le coût au $\mathrm{m}^{3} / \mathrm{s}$ des déversoirs est élevé pour la crue de sûreté (directement pour les barrages vannés, indirectement par le stockage perdu des seuils libres). Le coût au $\mathrm{m}^{3} / \mathrm{s}$ peut être beaucoup plus faible pour des débits excédentaires jusqu'à la crue de danger de rupture, car on admet alors un réservoir plus haut et la possibilité de quelques dommages ou pertes : les solutions correspondantes incluent l'aménagement de la crête de remblais, l'emploi de fusibles, l'association de deux déversoirs, ...

Il peut donc être intéressant, tout en choisissant une très faible probabilité pour la crue de danger de rupture (qui correspond à la sécurité réelle) d'admettre une plus grande probabilité qu'en France pour la crue de sûreté (par exemple une crue voisine de la crue centennale). Le débit de cette crue de sûreté ne sera souvent qu'un tiers du débit de la crue de danger de rupture et l'économie peut être très importante.

La crue centennale peut être bien évaluée par les méthodes probabilistes (largement développées en France). La crue de danger de rupture, avec une probabilité de l'ordre de $10^{-5}$ ou la P.M.F. est beaucoup plus difficile à évaluer, les méthodes probabilistes et déterministes pouvant être utilisées simultanément. Pour les bassins versants inférieurs à $1000 \mathrm{~km}^{2}$, une comparaison avec les maxima mondiaux $\left(700 \mathrm{~m}^{3} / \mathrm{s}\right.$ pour $10 \mathrm{~km}^{2}$ et $3000 \mathrm{~m}^{3} / \mathrm{s}$ pour $100 \mathrm{~km}^{2}$ ) peut être la base d'une évaluation complémentaire facile. On peut multiplier le maximum mondial pour une même surface de bassin par le ratio entre les pluies régionales maximales enregistrées en quelques heures et les pluies maximales mondiales de même durée et faire quelques ajustements mineurs pour tenir compte des autres caractéristiques du bassin. Limitée aux crues extrêmes et aux bassins versants de taille modérée dans les zones de pluies intenses, cette méthode simpliste n'est peutêtre pas moins fiable que d'autres ; pour de telles crues, la nature du terrain a en effet peu d'importance.

\section{CONCLUSIONS}

Nous proposons une terminologie et des définitions associées qui nous semblent de nature à préciser et clarifier la pratique actuelle, y compris dans le cas des ouvrages de protection contre les crues. 
Par ailleurs, nous proposons de mettre en avant les notions de cotes de projet, plutôt que celle de crues de projet. Ceci est particulièrement intéressant vu que l'on dispose désormais de méthodes permettant d'obtenir la distribution de fréquence des cotes atteintes dans la retenue.

1. Pour la prédétermination des crues extrêmes, on dispose maintenant de nouvelles méthodes de simulation d'événements pluviométriques et de modèles pluies-débits à faible pas de temps qui permettent de générer de longues chroniques de crues reproduisant la diversité des hydrogrammes que l'on peut observer dans la réalité (crues courtes mais pointues, crues étalées mais plus volumineuses, crues à pics multiples). Il est ainsi possible de simuler le passage de ces crues sur le barrage, puis d'établir une distribution de fréquence des cotes de remplissage en crue.

2. Il est également possible d'intégrer la variabilité de la cote de la retenue au moment de l'arrivée de la crue, en calant une loi statistique (si possible saisonnière) sur les courbes de remplissage de la retenue. Couplée à une étude saisonnière des crues, la méthode consiste à tirer aléatoirement par simulations de Monte Carlo des couples d'hydrogrammes et de cotes initiales et à procéder au calcul du laminage de la crue par la retenue pour obtenir une cote. On peut ensuite établir une distribution de fréquence de ces cotes.

3. Les méthodes d'étude de la sûreté de fonctionnement sont maintenant opérationnelles pour être appliquées aux évacuateurs de crues, en vue de définir les situations de défaillance, de les associer le cas échéant à l'événement incident et de classer ces combinaisons en fonction de leurs probabilités d'occurrence. Cela ouvre des perspectives dans le cadre des études de danger et constituerait un progrès pour l'étude du risque crues des dossiers PPI, où l'on ne considère aujourd'hui que la situation de non-fonctionnement de la vanne de plus grande capacité.

Au final, il paraît donc possible d'associer des probabilités d'occurrence au dépassement de la cote de sûreté ou de la cote de danger de rupture. Parallèlement, les autorités de contrôle et les instances professionnelles pourraient fixer des valeurs minimales cibles pour ces probabilités, en fonction des types d'ouvrages, et de leurs classes. Ces propositions ouvrent également des perspectives pour les études de dangers désormais obligatoires pour les barrages de classes $\mathrm{A}$ et $\mathrm{B}$ et pour les digues de classes $\mathrm{A}$ à $\mathrm{C}$.

\section{REFERENCES}

\section{[1] CIGB ICOLD (1992). Selection of Design Flood. Bulletin 82, Paris}

[2]Degoutte, G., coordinateur. (1997). Petits barrages ; recommandations pour la conception, la réalisation et le suivi. CFGB, Cemagref éditions, 176 p.

[3] CIGB ICOLD (2003). Dams and Floods - Guidelines and cases histories. Bulletin 125, Paris

[4] Goutx, D., Tourment, R., Mériaux, P. (2005). Éléments de conception hydraulique des déversoirs des endiguements de protection contre les inondations. Ingénieries - E AT, $\mathrm{n}^{\circ}$ Spécial Sécurité des digues fluviales et de navigation, p. 169 - 178

[5]Lempérière, F., Vigny, J.-P. (2005). Should we forget the traditional design flood? Hydropower and Dams - Issue 2

[6] Lavabre, J., Arnaud, P., Royet, P., Finé, J. A., Delichère, S., Fang, Z. X., Foussard, F. (2009) Crues de projet ou cotes de projet? Exemple des barrages écrêteurs de crue du département du Gard. Colloque CFBR-SHF: «Dimensionnement et fonctionnement des évacuateurs de crues», 20-21 janvier 2009, Lyon

[7] Peyras, L., Carvajal, C., Arnaud, P., Royet, P., Boissier, D. (2009) Modélisation probabiliste de la cote de remplissage d'un barrage. Colloque CFBR-SHF: «Dimensionnement et fonctionnement des évacuateurs de crues», 20-21 janvier 2009, Lyon 